\title{
Operations of Points on Elliptic Curve in Affine Coordinates ${ }^{1}$
}

\author{
Yuichi Futa \\ Tokyo University of Technology \\ Tokyo, Japan \\ Yasunari Shidama \\ Shinshu University \\ Nagano, Japan
}

Hiroyuki Okazaki

Shinshu University

Nagano, Japan

\begin{abstract}
Summary. In this article, we formalize in Mizar 1, 2] a binary operation of points on an elliptic curve over $\mathbf{G F}(\mathbf{p})$ in affine coordinates. We show that the operation is unital, complementable and commutative. Elliptic curve cryptography [3], whose security is based on a difficulty of discrete logarithm problem of elliptic curves, is important for information security.
\end{abstract}

MSC: 14H52 14K05 68T99 03B35

Keywords: elliptic curve; commutative operation

MML identifier: EC_PF_3, version: 8.1.09 5.59.1363

\section{Set of Points on Elliptic Curve in Affine Coordinates}

From now on $p$ denotes a 5 or greater prime number and $z$ denotes an element of the parameters of elliptic curve $p$.

Now we state the propositions:

(1) Let us consider a prime number $p$, elements $a, b$ of $\mathrm{GF}(p)$, and an element $P$ of $\operatorname{ProjCo}(\mathrm{GF}(p))$. Suppose $P=\langle 0,1,0\rangle$ or $(P)_{3,3}=1$. Then the represent point of $P=P$.

${ }^{1}$ This work was supported by JSPS KAKENHI Grant Numbers JP15K00183 and JP17K00182. 
Proof: If $P=\langle 0,1,0\rangle$, then the represent point of $P=P$. If $(P)_{3,3}=1$, then the represent point of $P=P$ by [5, (2)], [6, (3)].

(2) Let us consider a 5 or greater prime number $p$, an element $z$ of the parameters of elliptic curve $p$, and elements $P, O$ of $\operatorname{EC}_{\text {SetProjCo }}\left((z)_{1}\right)$. Suppose $O=\langle 0,1,0\rangle$. Then $(P)_{3,3}=0$ if and only if $P \equiv O$. The theorem is a consequence of (1).

(3) Let us consider a 5 or greater prime number $p$, an element $z$ of the parameters of elliptic curve $p$, and an element $P$ of $\operatorname{EC}_{\text {SetProjCo }}\left((z)_{1}\right)$. If $(P)_{\mathbf{3}, 3}=0$, then $P \equiv\left(\right.$ compell $\left._{\mathrm{ProjCo}}(z, p)\right)(P)$. The theorem is a consequence of $(2)$.

(4) Let us consider elements $P, O$ of $\operatorname{EC}_{\text {SetProjCo }}\left((z)_{1}\right)$. Suppose $O=\langle 0$, $1,0\rangle$. Then $\left(\operatorname{addell}_{\mathrm{ProjCo}}(z, p)\right)\left(P,\left(\operatorname{compell}_{\mathrm{ProjCo}}(z, p)\right)(P)\right) \equiv O$. The theorem is a consequence of (2) and (3).

Let $p$ be a 5 or greater prime number and $z$ be an element of the parameters of elliptic curve $p$. The functor EC-SetAffCo $(z, p)$ yielding a non empty subset of $\mathrm{EC}_{\mathrm{SetProjCo}}\left((z)_{\mathbf{1}}\right)$ is defined by the term

(Def. 1) $\left\{P\right.$, where $P$ is an element of $\operatorname{EC}_{\text {SetProjCo }}\left((z)_{1}\right):(P)_{3,3}=1$ or $P=\langle 0$, $1,0\rangle\}$.

Now we state the proposition:

(5) $\langle 0,1,0\rangle$ is an element of EC-SetAffCo $(z, p)$.

Let us consider a 5 or greater prime number $p$, an element $z$ of the parameters of elliptic curve $p$, and an element $P$ of $\operatorname{EC}_{\text {SetProjCo }}\left((z)_{1}\right)$. Now we state the propositions:

(6) The represent point of $P$ is an element of $\operatorname{EC}-\operatorname{SetAffCo}(z, p)$.

(7) If $P \in$ EC-SetAffCo $(z, p)$, then the represent point of $P=P$. The theorem is a consequence of (1).

Let us consider elements $P, O$ of $\operatorname{EC}_{\mathrm{SetProjCo}}\left((z)_{1}\right)$. Now we state the propositions:

(8) If $O=\langle 0,1,0\rangle$ and $P \not \equiv O$, then (the represent point of $P)_{\mathbf{3}, 3}=1$. The theorem is a consequence of $(2)$.

(9) Suppose $O=\langle 0,1,0\rangle$ and the represent point of $P \equiv O$. Then

(i) the represent point of $P=O$, and

(ii) $P \equiv O$.

The theorem is a consequence of (2) and (1).

(10) Let us consider an element $P$ of $\operatorname{ProjCo}(\mathrm{GF}(p))$. Then the represent point of the represent point of $P=$ the represent point of $P$. The theorem is a consequence of (1). 
(11) Let us consider elements $P, Q$ of $\operatorname{EC}_{\text {SetProjCo }}\left((z)_{1}\right)$. Suppose the represent point of $P \equiv$ the represent point of $Q$. Then the represent point of $P=$ the represent point of $Q$. The theorem is a consequence of (10).

Let $p$ be a 5 or greater prime number and $z$ be an element of the parameters of elliptic curve $p$. The functor compell- $\operatorname{AffCo}(z, p)$ yielding a unary operation on $\mathrm{EC}-\operatorname{Set} \operatorname{AffCo}(z, p)$ is defined by

(Def. 2) for every element $P$ of EC-SetAffCo $(z, p)$, it $(P)=$ the represent point of $\left(\right.$ compell $\left._{\text {ProjCo }}(z, p)\right)(P)$.

Let $F$ be a function from EC-SetAffCo $(z, p)$ into $\operatorname{EC-SetAffCo}(z, p)$ and $P$ be an element of EC-SetAffCo $(z, p)$. Let us observe that the functor $F(P)$ yields an element of EC-SetAffCo $(z, p)$. The functor addell-AffCo $(z, p)$ yielding a binary operation on EC-SetAffCo $(z, p)$ is defined by

(Def. 3) for every elements $P, Q$ of EC-SetAffCo $(z, p)$, it $(P, Q)=$ the represent point of $\left(\right.$ addell $\left._{\operatorname{ProjCo}}(z, p)\right)(P, Q)$.

Let $F$ be a function from EC-SetAffCo $(z, p) \times \operatorname{EC}-\operatorname{SetAffCo}(z, p)$ into

EC-SetAffCo $(z, p)$ and $Q, R$ be elements of EC-SetAffCo $(z, p)$. Let us observe that the functor $F(Q, R)$ yields an element of EC-SetAffCo $(z, p)$. Now we state the proposition:

(12) Let us consider elements $P, O$ of $\operatorname{EC}_{\mathrm{SetProjCo}}\left((z)_{1}\right)$. Suppose $O=\langle 0,1$, $0\rangle$. Then

(i) $\left(\operatorname{addell}_{\mathrm{ProjCo}}(z, p)\right)(P, O) \equiv P$, and

(ii) $\left(\right.$ addell $\left._{\mathrm{ProjCo}}(z, p)\right)(O, P) \equiv P$.

Let us consider elements $P, O$ of EC-SetAffCo $(z, p)$. Now we state the propositions:

(13) If $O=\langle 0,1,0\rangle$, then (addell-AffCo $(z, p))(O, P)=P$. The theorem is a consequence of (12) and (7).

(14) If $O=\langle 0,1,0\rangle$, then (addell-AffCo $(z, p))(P, O)=P$. The theorem is a consequence of (12) and (7).

(15) Let us consider an element $O$ of EC-SetAffCo $(z, p)$. Suppose $O=\langle 0,1$, $0\rangle$. Then $O$ is a unity w.r.t. addell- $\operatorname{AffCo}(z, p)$. The theorem is a consequence of (13) and (14).

(16) Let us consider elements $P, O$ of EC-SetAffCo $(z, p)$. Suppose $O=\langle 0,1$, $0\rangle$. Then (addell-AffCo $(z, p))(P,(\operatorname{compell-AffCo}(z, p))(P))=O$. The theorem is a consequence of $(7),(4)$, and (2). 


\section{Commutative Property of Operations of Points on Elliptic Curve}

Now we state the propositions:

(17) Let us consider a 5 or greater prime number $p$, an element $z$ of the parameters of elliptic curve $p$, and elements $P, Q, O, P_{3}, Q_{3}$ of $\mathrm{EC}_{\text {SetProjCo }}\left((z)_{1}\right)$. Suppose $O=\langle 0,1,0\rangle$ and $P \not \equiv O$ and $Q \not \equiv O$ and $P \not \equiv Q$. Suppose $P_{3}=\left(\operatorname{addell}_{\mathrm{ProjCo}}(z, p)\right)(P, Q)$ and $Q_{3}=\left(\operatorname{addell}_{\mathrm{ProjCo}}(z, p)\right)(Q, P)$. Then

(i) $\left(Q_{3}\right)_{\mathbf{1}, 3}=-\left(P_{3}\right)_{\mathbf{1}, 3}$, and

(ii) $\left(Q_{3}\right)_{2,3}=-\left(P_{3}\right)_{2,3}$, and

(iii) $\left(Q_{3}\right)_{3,3}=-\left(P_{3}\right)_{3,3}$.

Proof: Reconsider $g_{2}=2 \bmod p$ as an element of $\operatorname{GF}(p)$. Set $g f_{1 P Q}=$ $(Q)_{\mathbf{2}, 3} \cdot\left((P)_{\mathbf{3}, 3}\right)-(P)_{\mathbf{2}, 3} \cdot\left((Q)_{\mathbf{3}, 3}\right)$. Set $g f_{2 P Q}=(Q)_{\mathbf{1}, 3} \cdot\left((P)_{\mathbf{3}, 3}\right)-(P)_{\mathbf{1}, 3} \cdot$ $\left((Q)_{3,3}\right)$. Set $g f_{3 P Q}=g f_{1 P Q}{ }^{2} \cdot\left((P)_{3,3}\right) \cdot\left((Q)_{3,3}\right)-g f_{2 P Q}{ }^{3}-g_{2} \cdot\left(g f_{2 P Q}{ }^{2}\right)$. $\left((P)_{\mathbf{1}, 3}\right) \cdot\left((Q)_{\mathbf{3}, 3}\right)$. Set $g f_{1 Q P}=(P)_{\mathbf{2}, 3} \cdot\left((Q)_{\mathbf{3}, 3}\right)-(Q)_{\mathbf{2}, 3} \cdot\left((P)_{\mathbf{3}, 3}\right)$. Set $g f_{2 Q P}=(P)_{\mathbf{1}, 3} \cdot\left((Q)_{\mathbf{3}, 3}\right)-(Q)_{\mathbf{1}, 3} \cdot\left((P)_{\mathbf{3}, 3}\right)$. Set $g f_{3 Q P}=g f_{1 Q P}{ }^{2} \cdot\left((Q)_{\mathbf{3}, 3}\right)$. $\left((P)_{\mathbf{3}, 3}\right)-g f_{2 Q P}{ }^{3}-g_{2} \cdot\left(g f_{2 Q P}{ }^{2}\right) \cdot\left((Q)_{1,3}\right) \cdot\left((P)_{3,3}\right) \cdot g f_{3 Q P}=g f_{3 P Q} \cdot\left(Q_{3}\right)_{\mathbf{1}, 3}=$ $-\left(P_{3}\right)_{\mathbf{1}, 3} \cdot\left(Q_{3}\right)_{\mathbf{2}, 3}=-\left(P_{3}\right)_{\mathbf{2}, 3} \cdot\left(Q_{3}\right)_{\mathbf{3}, 3}=-\left(P_{3}\right)_{\mathbf{3}, 3}$.

(18) Let us consider elements $P, Q, O, P_{3}, Q_{3}$ of $\operatorname{EC}_{\text {SetProjCo }}\left((z)_{1}\right)$, and an element $d$ of $\operatorname{GF}(p)$. Suppose $O=\langle 0,1,0\rangle$ and $d \neq 0_{\mathrm{GF}(p)}$ and $(Q)_{\mathbf{1}, 3}=d \cdot\left((P)_{\mathbf{1}, 3}\right)$ and $(Q)_{\mathbf{2}, 3}=d \cdot\left((P)_{\mathbf{2}, 3}\right)$ and $(Q)_{\mathbf{3}, 3}=d \cdot\left((P)_{\mathbf{3}, 3}\right)$ and $P \not \equiv O$ and $Q \not \equiv O$ and $P \equiv Q$ and $P_{3}=\left(\operatorname{addell}_{\operatorname{ProjCo}}(z, p)\right)(P, Q)$ and $Q_{3}=\left(\right.$ addell $\left._{\text {ProjCo }}(z, p)\right)(Q, P)$. Then

(i) $\left(Q_{3}\right)_{\mathbf{1}, 3}=d^{6} \cdot\left(\left(P_{3}\right)_{1,3}\right)$, and

(ii) $\left(Q_{3}\right)_{\mathbf{2}, 3}=d^{6} \cdot\left(\left(P_{3}\right)_{\mathbf{2}, 3}\right)$, and

(iii) $\left(Q_{3}\right)_{\mathbf{3}, 3}=d^{6} \cdot\left(\left(P_{3}\right)_{3,3}\right)$.

(19) Let us consider elements $P, Q$ of $\operatorname{EC}_{\text {SetProjCo }}\left((z)_{1}\right)$.

Then $\left(\operatorname{addell}_{\text {ProjCo }}(z, p)\right)(P, Q) \equiv\left(\operatorname{addell}_{\operatorname{ProjCo}}(z, p)\right)(Q, P)$. The theorem is a consequence of (17) and (18).

(20) Let us consider elements $P, Q$ of EC-SetAffCo $(z, p)$.

Then (addell-AffCo $(z, p))(P, Q)=($ addell-AffCo $(z, p))(Q, P)$. The theorem is a consequence of (19).

Let $p$ be a 5 or greater prime number and $z$ be an element of the parameters of elliptic curve $p$. One can verify that addell- $\operatorname{AffCo}(z, p)$ is non empty, commutative, and unital.

The functor 0 -EC $(z, p)$ yielding an element of $\operatorname{EC-SetAffCo}(z, p)$ is defined by the term 
(Def. 4) $\langle 0,1,0\rangle$.

Let us consider $p$ and $z$. Let us observe that $\langle\mathrm{EC}-\operatorname{SetAffCo}(z, p)$, addell-AffCo $(z, p)\rangle$ is Abelian and $\langle\mathrm{EC}-\operatorname{Set} \operatorname{AffCo}(z, p)$, addell- $\operatorname{AffCo}(z, p), 0-\mathrm{EC}(z, p)\rangle$ is left zeroed and right zeroed and $\langle\mathrm{EC}-\operatorname{Set} A f f C o(z, p)$, addell-AffCo $(z, p), 0-\mathrm{EC}(z, p)\rangle$ is complementable.

Let $p$ be a 5 or greater prime number and $z$ be an element of the parameters of elliptic curve $p$. One can verify that $\langle\operatorname{EC}-\operatorname{Set} \operatorname{AffCo}(z, p)$, addell-AffCo $(z, p)\rangle$ is unital.

Now we state the proposition:

(21) Let us consider a 5 or greater prime number $p$, and an element $z$ of the parameters of elliptic curve $p$. Then $\mathbf{1}_{\langle\operatorname{EC}-\operatorname{SetAffCo}(z, p), \operatorname{addell}-\operatorname{AffCo}(z, p)\rangle}=$ 0 -EC $(z, p)$. The theorem is a consequence of (15).

Let $p$ be a 5 or greater prime number and $z$ be an element of the parameters of elliptic curve $p$. One can check that $\langle\operatorname{EC-SetAffCo}(z, p)$, addell-AffCo $(z, p)\rangle$ is commutative, group-like, and non empty.

Now we state the propositions:

(22) Let us consider elements $P_{1}, P_{2}, Q$ of $\mathrm{EC}_{\text {SetProjCo }}\left((z)_{\mathbf{1}}\right)$. Suppose $P_{1} \equiv$ $P_{2}$. Then $\left(\operatorname{addell}_{\operatorname{ProjCo}}(z, p)\right)\left(P_{1}, Q\right) \equiv\left(\operatorname{addell}_{\mathrm{ProjCo}}(z, p)\right)\left(P_{2}, Q\right)$. The theorem is a consequence of (19).

(23) Let us consider elements $P, Q_{1}, Q_{2}$ of $\operatorname{EC}_{\text {SetProjCo }}\left((z)_{1}\right)$. Suppose $Q_{1} \equiv$ $Q_{2}$. Then $\left(\operatorname{addell}_{\mathrm{ProjCo}}(z, p)\right)\left(P, Q_{1}\right) \equiv\left(\operatorname{addell}_{\mathrm{ProjCo}}(z, p)\right)\left(P, Q_{2}\right)$. The theorem is a consequence of (19) and (22).

(24) Let us consider elements $P_{1}, P_{2}, Q_{1}, Q_{2}$ of $\mathrm{EC}_{\text {SetProjCo }}\left((z)_{1}\right)$. Suppose $P_{1} \equiv P_{2}$ and $Q_{1} \equiv Q_{2}$. Then $\left(\right.$ addell $\left._{\operatorname{ProjCo}}(z, p)\right)\left(P_{1}, Q_{1}\right) \equiv$ (addell $\left._{\text {ProjCo }}(z, p)\right)\left(P_{2}, Q_{2}\right)$. The theorem is a consequence of $(22)$ and $(23)$.

(25) Let us consider elements $P, O$ of $\mathrm{EC}_{\text {SetProjCo }}\left((z)_{1}\right)$. Suppose $O=\langle 0,1$, $0\rangle$. Then $P \equiv O$ if and only if $\left(\operatorname{compell}_{\mathrm{ProjCo}}(z, p)\right)(P) \equiv O$.

(26) Let us consider elements $P, Q$ of $\mathrm{EC}_{\text {SetProjCo }}\left((z)_{1}\right)$, and an element $a$ of $\mathrm{GF}(p)$. Suppose $a \neq 0_{\mathrm{GF}(p)}$ and $(P)_{\mathbf{1}, 3}=a \cdot\left((Q)_{\mathbf{1}, 3}\right)$ and $(P)_{\mathbf{2}, 3}=$ $a \cdot\left((Q)_{\mathbf{2}, 3}\right)$ and $(P)_{\mathbf{3}, 3}=a \cdot\left((Q)_{\mathbf{3}, 3}\right)$. Then $P \equiv Q$.

(27) Let us consider elements $P, Q$ of $\mathrm{EC}_{\text {SetProjCo }}\left((z)_{1}\right)$, and elements $g_{2}$, $g f_{1}, g f_{2}, g f_{3}$ of $\operatorname{GF}(p)$. Suppose $P \not \equiv Q$ and $(P)_{\mathbf{3}, 3}=1$ and $(Q)_{\mathbf{3}, 3}=1$ and $g_{2}=2 \bmod p$ and $g f_{1}=(Q)_{\mathbf{2}, 3}-(P)_{\mathbf{2}, 3}$ and $g f_{2}=(Q)_{\mathbf{1}, 3}-(P)_{\mathbf{1}, 3}$ and $g f_{3}=g f_{1}^{2}-g f_{2}{ }^{3}-g_{2} \cdot\left(g f_{2}^{2}\right) \cdot\left((P)_{1,3}\right)$. Then $\left(\operatorname{addell}_{\operatorname{ProjCo}}(z, p)\right)(P, Q)=$ $\left\langle g f_{2} \cdot g f_{3}, g f_{1} \cdot\left(g f_{2}{ }^{2} \cdot\left((P)_{\mathbf{1}, 3}\right)-g f_{3}\right)-g f_{2}{ }^{3} \cdot\left((P)_{\mathbf{2}, 3}\right), g f_{2}{ }^{3}\right\rangle$. The theorem is a consequence of $(2)$.

(28) Let us consider elements $P, Q$ of $\mathrm{EC}_{\text {SetProjCo }}\left((z)_{1}\right)$, and elements $g_{2}$, $g_{3}, g_{4}, g_{8}, g f_{1}, g f_{2}, g f_{3}, g f_{4}$ of $\mathrm{GF}(p)$. Suppose $P \equiv Q$ and $(P)_{3,3}=1$ and $(Q)_{3,3}=1$ and $g_{2}=2 \bmod p$ and $g_{3}=3 \bmod p$ and $g_{4}=4 \bmod p$ and $g_{8}=$ 
$8 \bmod p$ and $g f_{1}=(z)_{\mathbf{1}}+g_{3} \cdot\left(\left((P)_{\mathbf{1}, 3}\right)^{2}\right)$ and $g f_{2}=(P)_{\mathbf{2}, 3}$ and $g f_{3}=(P)_{\mathbf{1}, 3}$. $\left((P)_{2,3}\right) \cdot g f_{2}$ and $g f_{4}=g f_{1}^{2}-g_{8} \cdot g f_{3}$. Then $\left(\operatorname{addell}_{\mathrm{ProjCo}}(z, p)\right)(P, Q)=$ $\left\langle g_{2} \cdot g f_{4} \cdot g f_{2}, g f_{1} \cdot\left(g_{4} \cdot g f_{3}-g f_{4}\right)-g_{8} \cdot\left(\left((P)_{2,3}\right)^{2}\right) \cdot\left(g f_{2}^{2}\right), g f_{8} \cdot\left(g f_{2}{ }^{3}\right)\right\rangle$. The theorem is a consequence of $(2)$.

Let us consider elements $P, Q$ of $\mathrm{EC}_{\mathrm{SetProjCo}}\left((z)_{\mathbf{1}}\right)$. Now we state the propositions:

(29) Suppose $(P)_{\mathbf{3}, 3}=1$ and $(Q)_{\mathbf{3}, 3}=1$.

Then $\left(\operatorname{compell}_{\mathrm{ProjCo}}(z, p)\right)\left(\left(\operatorname{addell}_{\mathrm{ProjCo}}(z, p)\right)(P, Q)\right) \equiv\left(\operatorname{addell}_{\mathrm{ProjCo}}(z, p)\right)$

$\left(\left(\operatorname{compell}_{\mathrm{ProjCo}}(z, p)\right)(P),\left(\operatorname{compell}_{\mathrm{ProjCo}}(z, p)\right)(Q)\right)$. The theorem is a consequence of $(27),(28)$, and (26).

(30) $\left(\operatorname{compell}_{\mathrm{ProjCo}}(z, p)\right)\left(\left(\operatorname{addell}_{\mathrm{ProjCo}}(z, p)\right)(P, Q)\right) \equiv\left(\operatorname{addell}_{\mathrm{ProjCo}}(z, p)\right)$

$\left(\left(\operatorname{compell}_{\mathrm{ProjCo}}(z, p)\right)(P),\left(\operatorname{compell}_{\mathrm{ProjCo}}(z, p)\right)(Q)\right)$. The theorem is a consequence of (25), (8), (29), (24), and (2).

(31) Let us consider elements $P, O$ of $\mathrm{EC}_{\text {SetProjCo }}\left((z)_{1}\right)$. Suppose $O=\langle 0,1$, $0\rangle$ and $P \not \equiv O$. Then $(P)_{\mathbf{2}, 3}=0_{\mathrm{GF}(p)}$ if and only if

$\left(\operatorname{addell}_{\mathrm{ProjCo}}(z, p)\right)(P, P) \equiv O$.

ProOF: Reconsider $g_{8}=8 \bmod p$ as an element of $\operatorname{GF}(p)$.

$\left(\left(\operatorname{addell}_{\mathrm{ProjCo}}(z, p)\right)(P, P)\right)_{\mathbf{3}, 3}=0 . g_{8} \neq 0_{\mathrm{GF}(p)} \cdot(P)_{\mathbf{3}, 3} \neq 0$ by [4, (23)], [5, $(28)]$.

\section{REFERENCES}

[1] Grzegorz Bancerek, Czesław Byliński, Adam Grabowski, Artur Korniłowicz, Roman Matuszewski, Adam Naumowicz, Karol Pakk, and Josef Urban. Mizar: State-of-the-art and beyond. In Manfred Kerber, Jacques Carette, Cezary Kaliszyk, Florian Rabe, and Volker Sorge, editors, Intelligent Computer Mathematics, volume 9150 of Lecture Notes in Computer Science, pages 261-279. Springer International Publishing, 2015. ISBN 978-3319-20614-1. doi 10.1007/978-3-319-20615-8_17.

[2] Grzegorz Bancerek, Czesław Byliński, Adam Grabowski, Artur Korniłowicz, Roman Matuszewski, Adam Naumowicz, and Karol Pąk. The role of the Mizar Mathematical Library for interactive proof development in Mizar. Journal of Automated Reasoning, 61(1):9-32, 2018. do1: $10.1007 /$ s10817-017-9440-6

[3] I. Blake, G. Seroussi, and N. Smart. Elliptic Curves in Cryptography. Number 265 in London Mathematical Society Lecture Note Series. Cambridge University Press, 1999.

[4] Yuichi Futa, Hiroyuki Okazaki, and Yasunari Shidama. Set of points on elliptic curve in projective coordinates. Formalized Mathematics, 19(3):131-138, 2011. doi:10.2478/v10037011-0021-6.

[5] Yuichi Futa, Hiroyuki Okazaki, Daichi Mizushima, and Yasunari Shidama. Operations of points on elliptic curve in projective coordinates. Formalized Mathematics, 20(1):87-95, 2012. doi $10.2478 / \mathrm{v} 10037-012-0012-2$.

[6] Artur Korniłowicz. Recursive definitions. Part II Formalized Mathematics, 12(2):167-172, 2004. 